\title{
Correlation of Visceral Obesity and Interleukin-6 Level on LUTS Due to Benign Prostatic Enlargement
}

\section{Bambang Noegroho (iD) Safendra Siregar \\ Albert Ivan Simangunsong}

Department of Urology, Faculty of Medicine Universitas Padjadjaran/Hasan Sadikin Hospital, Bandung, Indonesia
Correspondence: Bambang Noegroho Email bsn.urologi@gmail.com
Background: Benign prostatic enlargement (BPE) is a common condition in older men causing lower urinary tract symptoms (LUTS). Numerous studies have shown correlations of obesity and age as risk factors of LUTS due to BPE. However, there are only a few studies showing the increase of interleukin-6 (IL-6) as a risk factor in LUTS. IL-6 expression is regulated in the prostate. IL-6 was found to be increased in several conditions, such as infection, inflammation, and obesity. The aim of this study is to correlate visceral obesity and IL-6 expression in the prostate with BPE and LUTS.

Methods: We analyzed the correlation of the Visceral Adiposity Index (VAI) and IL-6 of 52 patients with LUTS due to BPE. Statistical analysis was tested using an independent $t$-test. Data were analyzed with SPSS ver 20.1.

Results: Of 52 BPE subjects, who had not received any therapy, 16 patients presented with severe LUTS (30.77\%), while the remaining $69.23 \%$ had mild-moderate LUTS complaints. IL-6 examination showed that obese patients and severe LUTS had higher IL-6, with $p=0.032$ and 0.01 , respectively. Correlation analysis showed a weak correlation between IL-6 and obesity $(\mathrm{r}=0.179, p=0.02)$. Based on regression study, it was found that the risk for a patient with visceral obesity to develop severe LUTS is 5-times higher than normal $(p=0.017)$.

Conclusion: This study found a correlation between the degree of visceral obesity assessed by VAI and IL-6 levels in BPE patients. In addition, there is also a correlation between the degree of visceral obesity and IL-6 levels on LUTS severity in people with BPE.

Keywords: benign prostatic enlargement, interleukin 6, lower urinary tract symptoms, visceral obesity

\section{Introduction}

Benign prostatic enlargement (BPE) is a condition characterized by the development of hyperplastic nodules in the transition zone of the prostate, and enlargement of the entire prostate. This is a very common benign tumor among middle-aged and elderly men. ${ }^{1}$ The prevalence of BPE increases after the age of 40, with a prevalence of $8-60 \%$ at the age of 90 years. ${ }^{2}$ Statistical data of Hasan Sadikin Bandung General Hospital in 2012-2016 showed that the mean age of BPE patients was 67.9 years in 718 cases. $^{3}$

According to the World Health Organization (WHO), obesity is an excess of adipose tissue or an abnormal increase in fat tissue which might cause health problems. ${ }^{4}$ The increasing of body mass index was associated with excess weight 
in the abdominal area which increases the abdominal pressure. This condition results in increased bladder pressure and urethral mobility, contributing to urinary incontinence. ${ }^{5}$

Obesity could cause an increase of Interleukin-6 (IL-6), which is a pro-inflammatory cytokine that increases in both acute and chronic inflammatory conditions. ${ }^{6}$ This cytokine is the most dominant interleukin found in both stromal and epithelial of patients with BPE. IL-6 receptors were found in prostate tissue which aims to regulate cell growth. ${ }^{7}$ A study by Gotera et $\mathrm{al}^{6}$ revealed a significant increase in serum IL-6 levels in BPE patients compared to non-BPE patients in the same age group.

There are only a few studies which show the increase of Interleukin-6 (IL-6) as a risk factor in LUTS. The diversity of results regarding the association between visceral obesity and the incidence of prostate enlargement and LUTS prompted the authors to investigate the association of visceral obesity using the visceral adiposity index (VAI) in patients who were included in the inclusion criteria.

\section{Methods}

This was an observational analytic study with a crosssectional design with consecutive sampling with minimal sampling using rule of thumb method, 5-10-times the number of independent variables studied. This study was conducted in men aged $\geq 50$ years who sought medical treatment at an outpatient clinic between the JanuaryOctober 2020. The inclusion criteria were patients aged $\geq 50$ years, this being the first visit to the urology clinic to seek medical treatment, never had any BPE treatment before, and willing to be involved in this study. The exclusion criteria were past history of BPE; urological patients diagnosed with diabetes mellitus, alcohol dependence, hypothyroidism, autoimmune diseases, fractures, joint disease, infectious conditions, and liver disease; patients with other conditions and or diseases which could cause LUTS symptoms (for instance urethral strictures, bladder stones, urinary tract infections); and patients with severe cognitive impairment (dementia, psychiatric disorders) who were unable to complete the IPSS.

Anthropometric examinations used as an alternative method for assessing visceral fat were waist circumference (WC) and sagittal abdominal diameter (SAD), Waist-toHeight Ratio (WHtR), Waist-to-Thigh Ratio (WTR), conicity index (CI), and the Visceral Adiposity Index (VAI). VAI was used as an alternative parameter to assess visceral fat using anthropometric and metabolic parameters which was chosen because it is fairly accurate and reflects the production of other adipocytokines, increased lipolysis, and free fatty acids; factors that cannot be identified simply by examining BMI, abdominal circumference, triglycerides, and HDL separately. ${ }^{6}$

The IPSS questionnaire, that has been validated to the Indonesian version, was used to measure the LUTS symptoms degree $($ mild $=0-7$, moderate $=8-19$, and severe $=20$ $35){ }^{8}$ The prostate volume enlargement $(>30 \mathrm{~mL})$, which indicates BPE, was assessed by using prostate USG in all participants. Visceral obesity was diagnosed by using anthropometric examination with a VAI score $>2$. The examination was carried out by measuring abdominal circumference, body mass index, triglyceride levels, and HDL. Then these parameters were entered into the VAI formula so that the VAI score was obtained.

Interleukin 6 (IL-6) level was measured by taking a blood sample mixed with a special reagent, named Human IL-6 Quantikine HS ELISA KIT in Hasan Sadikin General Hospital Bandung. This serum sample should be stored at a stable temperature of $-20^{\circ} \mathrm{C}$. Serum was centrifuged 1,000-times with normal levels of 0.2-10 $\mathrm{pg} / \mathrm{mL}$. Then the serum was added to the microplate containing the IL-6 preparation which had been added with $100 \mu \mathrm{L}$ of RD1W diluent assay to each well.

This research has been submitted through the Urology Department and the Ethics Committee for Health Research and has received ethical clearance with the number: LB.02.01/X.6.5/171/2020.

The data used in this study were primary data obtained from the researcher's observation, then the data were collected and processed by using SPSS (Statistical Product and Service Solution). The data were then analyzed in two ways, namely univariable analysis (descriptive analysis) and bivariable analysis. The amount of risk in the bivariable analysis was expressed as crude OR with 95\% confidence interval (CI). The $p$-value was considered significant if $p<0.05$. The OR value was considered a risk factor or difficult prediction if $\mathrm{OR} \geq 1.00$. Logistic regression analysis was used to analyze the relationship between visceral obesity and BPE and LUTS. The IL-6 variable was first tested for its normality, if the data was normally distributed, the test was continued with the Independent $t$-test.

\section{Results}

From the results of the research, 52 respondents who suffered from BPE weere obtained. Patients who had LUTS complaints were grouped into mild-moderate 
(IPSS $<20$ ) and severe (IPSS $\geq 20$ ) groups. A total of 16 patients presented with severe LUTS complaints (30.77\%), while the remaining $69.23 \%$ had mild-moderate LUTS complaints. A total of 41 patients were classified into the visceral obesity group based on VAI calculations, and the remaining 11 were normal (Table 1).

Anthropometric examination of the patients revealed that the patient's mean height was $163.37 \mathrm{~cm}$, the patient's average body weight was $67.33 \mathrm{~kg}$, and the patient's average BMI was $25.22 \mathrm{~kg} / \mathrm{m}^{2}$. The mean IL level for 6 patients was $7.51 \mathrm{pg} / \mathrm{mL}$ (Table 1).

Before analyzng the data, a normality test was carried out by using the Kolmogorov Smirnov test to see the data distribution. Normally distributed data were tested using the independent $t$-test and abnormally distributed data were tested using the Whitney Mann test. Based on Table 2, the mean value of IL-6 in visceral obese patients was higher than normal people $(p=0.032)$.

Based on Table 3, it was shown that the average IL-6 level in severe LUTS patients was higher than in patients

Table I Subject Characteristics

\begin{tabular}{|c|c|c|}
\hline Variable & Mean士SD & $\mathbf{N}(\%)$ \\
\hline Age & $67.26 \pm 10.67$ & \\
\hline \multicolumn{3}{|l|}{ LUTS } \\
\hline Mild-moderate & & $36(69.23 \%)$ \\
\hline Severe & & $16(30.77 \%)$ \\
\hline \multicolumn{3}{|l|}{ Ultrasound study } \\
\hline Prostate volume (cc) & $52.59 \pm 27.33$ & \\
\hline Intravesical prostatic & $12.85 \pm 2.24$ & \\
\hline Protrusion $(\mathrm{mm})$ & & \\
\hline Bladder thickness (mm) & $2.75 \pm 1.18$ & \\
\hline \multicolumn{3}{|l|}{ VAl } \\
\hline Normal & & II (2I.15\%) \\
\hline Visceral obesity & & $4 \mathrm{I}(78.85 \%)$ \\
\hline Body Mass Index (BMI) & $25.22 \pm 4.37$ & \\
\hline IL-6 & $7.51 \pm 8.80$ & \\
\hline
\end{tabular}

Table 2 Difference of Mean IL-6 with Visceral Obesity in BPE Patients

\begin{tabular}{|l|l|c|}
\hline Variable & Mean \pm SD & -value \\
\hline Normal & $6.21 \pm 6.48$ & $0.032^{*}$ \\
Visceral Obesity & $7.89 \pm 9.41$ & \\
\hline
\end{tabular}

Note: *Significant statistically, $p<0.05$.
Table 3 Difference in Mean IL-6 on LUTS

\begin{tabular}{|c|c|c|c|}
\hline \multirow[t]{2}{*}{ Variable } & Severe LUTS & Mild-Moderate LUTS & \multirow[t]{2}{*}{$p$-value } \\
\hline & Mean士SD & Mean士SD & \\
\hline IL-6 & $13.29 \pm 14.18$ & $4.94 \pm 2.13$ & $0.0 I^{*}$ \\
\hline
\end{tabular}

Note: *Significant statistically, $p<0.05$.

who experienced mild-moderate LUTS and this difference was statistically significant $(p=0.01)$.

Furthermore, a correlation test was carried out between IL-6 levels and the VAI value. Based on the Spearman correlation test, a weak relationship $(\mathrm{r}=0.176)$ was found between the two variables $(p=0.02)$ (Table 3 and Figure 1$)$.

A regression test was performed to assess the relationship between visceral obesity and LUTS in BPE patients (Table 4). There were $35.7 \%$ of severe LUTS patients with visceral obesity and $64.3 \%$ of severe LUTS patients who were not obese. Patients with visceral obesity have 5-times greater risk of developing LUTS compared to patients who do not have visceral obesity. This shows that patients with visceral obesity have a 5-times greater chance of developing LUTS than patients who were not suffering from visceral obesity.

\section{Discussion}

Enlarged prostate is a common medical condition affecting older men. Based on epidemiological studies, $70 \%$ of male patients aged 60-69 years have an enlarged prostate, and this number possibly increases to $80 \%$ in patients over 70 years of age. ${ }^{8}$ Many hypotheses support the effect of obesity on the incidence of prostate enlargement., ${ }^{9,10}$

According to a study of 166 patients in Turkey, there was no strong association which determined the relationship between high body mass index as an indicator of metabolic syndrome and the increasing of LUTS symptoms indicated by IPSS score. ${ }^{7}$ This result was similar to this study, which revealed that there was no significant difference between BMI among LUTS group. Several epidemiological studies have shown the association between obesity and the incidence of LUTS. ${ }^{11}$ For instance, a cross-sectional analysis conducted by the NHANES (National Health and Nutrition Examination Survey) showed that LUTS tends to be severe in obese patients with a BMI above 25 in the age group over 60 years. ${ }^{12}$ In addition, a study by Giovannucci et al showed that abdominal circumference also had an impact on the incidence of LUTS. ${ }^{12}$ Uniquely, many studies have failed to demonstrate this correlation. ${ }^{13-15}$ The cause of this contradictory result has remained unclear, but it is possible 


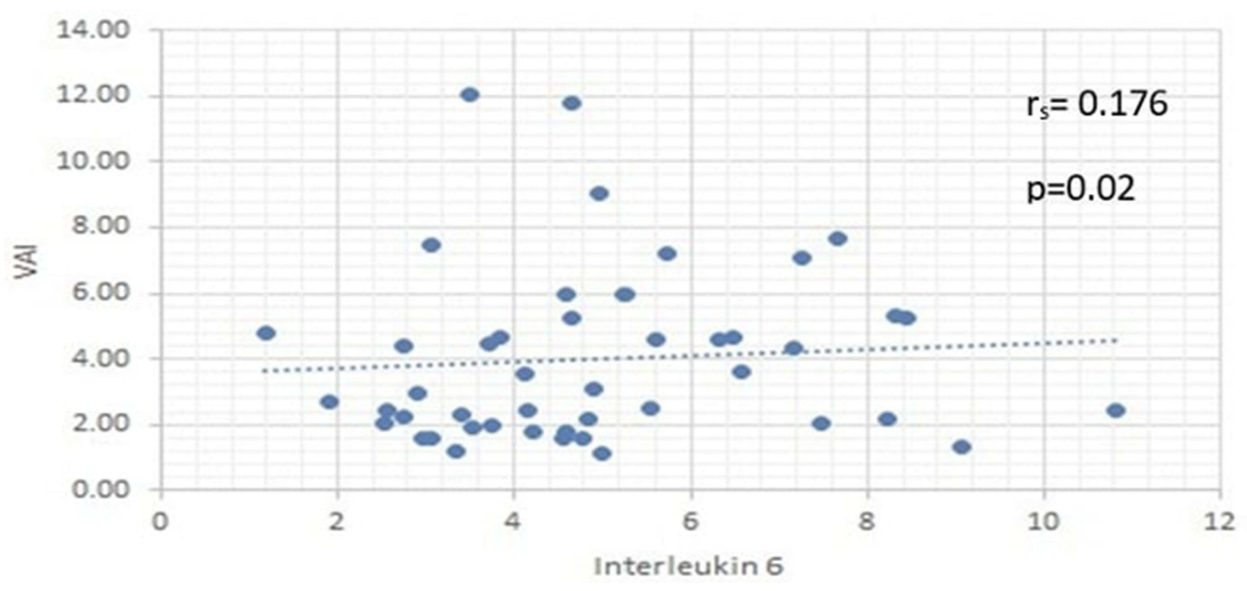

Figure I Scatter Plot graph of the relationship between IL-6 levels and VAI values.

that different methods of measuring the degree of LUTS, the onset of measurements and the presence of confounding factors such as inflammation might be the reason of this. ${ }^{15}$

Obesity is proposed to change hormonal conditions. An increased estrogen-androgen ratio occurs due to increasing enzyme P450 aromatase produced by fat tissue. Increased fat tissue, which means increasing the activity of this enzyme which will convert androgens into estrogen (testosterone into estradiol and androstenedione into estrone). ${ }^{15}$ Increased production of estradiol caused by the accumulation of fat mass, causes gonadotropin suppression and decreases testosterone production and causes a hypogonadal condition that supports prostate enlargement. ${ }^{16}$ In this study, there were no significant differences based on VAI measurements in BPE and non-BPE patients, but there were differences between patients with mild-moderate LUTS complaints and severe LUTS complaints.

The inflammatory process triggered by obesity causes micro-vascularization disorders, ischemia, oxidative stress, and promotes prostate enlargement. ${ }^{17,18}$ In obese patients, there was evidence of the increasing incidence of chronic inflammation caused by secretion of inflammatory cytokines by visceral fat. ${ }^{18}$

Obesity induces increasing levels of Interleukin-6 (IL-6), which is a pro-inflammatory cytokine. ${ }^{6}$ IL-6 receptors were

Table 4 Correlation Between IL-6 Levels and VAI Values in BPE Patients

\begin{tabular}{|c|c|c|c|}
\hline VAI & $\mathbf{r}_{\mathbf{s}}$ & $\boldsymbol{p}$-value & Conclusion \\
\hline IL-6 & 0.176 & $0.02 *$ & $\begin{array}{c}\text { A significant positive correlation was } \\
\text { found between IL-6 levels and VAI values }\end{array}$ \\
\hline
\end{tabular}

Note: *Significant statistically, $p<0.05$. found in prostate tissue which aims to regulate cell growth. ${ }^{7}$ In this study, there were significant differences in IL-6 levels between severe and mild-moderate LUTS patients. This supports several studies that show chronic inflammation plays a role in the occurrence of urinary complaints. In the elderly, chronic inflammation was statistically significant with the occurrence of complaints of nocturia, frequency, and urgency. A study by Robert et $a{ }^{19},{ }^{19}$ by using cytological and immunohistochemical parameters, showed that patients with inflammation had a higher IPSS score and a larger prostate volume. Through this study, we found a proposed mechanism that obesity is associated with elevated levels of IL-6 which would be a risk factor for LUTS, especially due to BPE. The limitation of this study is the small population, which cannot reflect the role of visceral obesity and IL-6 in the whole population.

This study found a correlation between the degree of visceral obesity as assessed by VAI and IL-6 levels in BPE patients. In addition, there is a correlation between the degree of visceral obesity and IL-6 levels on LUTS severity in people with BPE. A limitation of this study is that we did not examine IL-6 levels in the obese patient without LUTS.

\section{Data Sharing Statement}

All data generated or analyzed during this study can be requested from the corresponding author.

\section{Ethics Approval and Consent to Participate}

This research has been submitted through the Urology Department and the Ethics Committee for Health Research of RSHS Bandung and has received ethical 
clearance according the Declaration of Helsinki. Written informed consent for publication was obtained from all of the subject in this research.

\section{Consent for Publication}

Applicable.

\section{Funding}

There is no funding to report.

\section{Disclosure}

The authors reported no conflicts of interest for this work.

\section{References}

1. Zhao SC, Xia M, Tang JC. Associations between metabolik syndrome and clinical benign prostatic hyperplasia in a northern urban Han Chinese population: a prospective cohort study. Sci Rep. 2016;6:1-12. doi:10.1038/s41598-016-0001-8

2. Bin LK. Epidemiology of clinical benign prostatic hyperplasi. Asian J Urol. 2017;4(3):148-151. doi:10.1016/j.ajur.2017.06.004

3. Chaidir M, Rainy U. Pembesaran prostat jinak (Benign Prostatic Hyperplasia/BPE). 2015;8-33.

4. Aslan A, Çırakoğlu A, Kaşko Y. The relationship between body mass index and lower urinary tract symptoms in men. Middle Black Sea J Health Sci. 2019;5(August):138-144.

5. Shuster A, Patlas M, Pinthus JH, Mourtzakis M. The clinical importance of visceral adiposity: a critical review of methods for visceral adipose tissue analysis. Br J Radiol. 2012;85(1009):1-10. doi:10.1259/ bjr/38447238

6. Gotera W, Mahadita W, Bakta IM, et al. Waist circumference increased risk of benign prostatic hyperplasia through an increase in the level of interleukin-6 and insulin resistance in abdominal obesity patients. Bali Med J. 2017;6(1):204-210. doi:10.15562/bmj.v6i1.464

7. Dahle SE, Chokkalingam AP, Gao Y-T, et al. Body size and serum levels of insulin and leptin in relation to the risk of benign prostatic hyperplasia. $J$ Urol. 2002;168(2):599-604. doi:10.1016/S00225347(05)64687-3
8. Penson DF, Munro HM, Signorello LB, et al. Urologic diseases in America Project. Obesity, physical activity and lower urinary tract symptoms: results from the Southern Community Cohort Study. J Urol. 2011;186(6):2316-2322. doi:10.1016/j.juro.2011.07.067

9. Joseph MA, Harlow SD, Wei JT, et al. Risk factors for lower urinary tract symptoms in a population-based sample of African-American men. Am J Epidemiol. 2003;157:906. doi:10.1093/aje/kwg051

10. Wei JT, Calhoun E, Jacobsen SJ. Urologic diseases in America project: benign prostatic hyperplasia. J Urol. 2005;173:1256-1261. doi:10.1097/01.ju.0000155709.37840.fe

11. Monoarfa RA, Mochtar CA. Validation of Indonesian Version of IPSS. Indonesian J Urol. 2014;21(1):15-19.

12. Rohrmann S, Smit E, Giovannucci E, Platz EA. Associations of obesity with lower urinary tract symptoms and noncancer prostate surgery in the third national health and nutrition examination survey. Am J Epidemiol. 2004;159:390. doi:10.1093/aje/kwh060

13. Giovannucci E, Rimm EB, Chute CG, et al. Obesity and benign prostatic hyperplasia. Am J Epidemiol. 1994;140:989. doi:10.1093/ oxfordjournals.aje.a117206

14. Haidinger G, Temml C, Schatzl G, et al. Risk factorsfor lower urinary tract symptoms in elderly men. For the Prostate Study Group of the Austrian Society of Urology. Eur Urol. 2000;37:413. doi:10.1159/ 000020162

15. Wong SY, Woo J, Hong A, et al. Risk factors for lower urinary tract symptoms in southern Chinese men. Urology. 2006;68:1009. doi:10.1016/j.urology.2006.05.039

16. Williams G. Aromatase up-regulation, insulin and raised intracellular oestrogens in men, induce adiposity, metabolic syndrome and prostate disease, via aberrant ER-alpha and GPER signalling. Mol Cell Endocrinol. 2012;351:269-278. doi:10.1016/j.mce.2011.12.017

17. Parikesit D, Mochtar CA, Umbas R, et al. The impact of obesity towards prostate diseases. Prostate Int. 2016;4(1):1-6. doi:10.1016/j. prnil.2015.08.001

18. Penna G, Fibbi B, Amuchastegui S, et al. Human benign prostatic hyperplasia stromal cells as inducers and targets of chronic immuno-mediated inflammation. J Immunol. 2009;182:4056-4064. doi:10.4049/jimmunol.0801875

19. Samarinas M, Gravas S. The relationship between inflammation and LUTS/BPH. In: Lower Urinary Tract Symptoms and Benign Prostatic Hyperplasia. Academic Press; 2018:31-50.
Research and Reports in Urology

\section{Publish your work in this journal}

Research and Reports in Urology is an international, peer-reviewed, open access journal publishing original research, reports, editorials, reviews and commentaries on all aspects of adult and pediatric urology in the clinic and laboratory including the following topics: Pathology, pathophysiology of urological disease; Investigation and treatment of urological disease; Pharmacology of drugs used for the treatment of urological disease. The manuscript management system is completely online and includes a very quick and fair peer-review system, which is all easy to use. Visit http://www.dovepress.com/ testimonials.php to read real quotes from published authors. 